\title{
Perfil epidemiológico de idosas vinculadas a um clube de repouso
}

\author{
Epidemiologic Profile of Elderly Afilliated to a Nursing Home
}

\author{
Mara Moura Leal ${ }^{1}$, Ana Lourdes Avelar Nascimento ${ }^{1}$, Adelzir Malheiros e Silva Carvalho \\ Barbosa Haidar ${ }^{1}$, Maria Erivânia Alves de Araújo ${ }^{1}$, José Newton Lacet Vieira ${ }^{1}$
}

\begin{abstract}
Resumo: Descrever o perfil epidemiológico de idosas vinculadas a um clube de repouso. Tratou-se de um estudo descritivo e transversal. A população deste estudo foi composta por conveniência por 30 idosas com idade igual ou superior a 60 anos, vinculados ao clube de repouso Irmã Dulce em São Luis - MA, no período de março a junho de 2013. Foi aplicado um questionário para a identificação do perfil epidemiológico dessas idosas foi realizada uma entrevista, que permitiu verificar as doenças mais prevalentes, além de domínios vinculados a aspectos correlacionados a saúde mental, sensorial, físico, social, nutricional e assistência terapêutica. Os dados foram tabulados em uma planilha do programa Microsoft Office EXCEL 2007, e transferidos para análise no programa estatístico SPSS 18.0. As variáveis quantitativas foram descritas em média e desvio padrão média \pm $\mathrm{DP}$ e as variáveis qualitativas são apresentadas por meio de freqüências absolutas e percentuais. $A$ amostra apresentou uma população do sexo feminino $100 \%$ com média etária $73,2 \pm 8,14$. A análise estatística mostrou que $60 \%$ apresentaram duas ou mais patologias. Em relação a cognição $100 \%$ relataram boa cognição. Quanto à capacidade de realizar atividades de vida diária, todas $100 \%$ relataram ser independentes para a realização de atividades da vida diária. As idosas vinculadas a um clube de repouso apresentaram resultados satisfatórios para os itens cognição e a capacidade de realizar as atividades de vida diária. Porém, a maioria apresentou co-morbidades.
\end{abstract}

Palavras-chave: Epidemiologia. Idosas. Risco.

\begin{abstract}
To describe the epidemiological profile of elderly women members of a nursing home. Methods: This was a descriptive, cross-sectional, quantitative study. The study population was composed for convenience with 30 women, 60 years of age or more, affiliated to Sister Dulce nursing home, São Luís-MA, from March to June of 2013. A questionnaire was applied to identify the epidemiological profile of the elderly women affiliated to the nursing home. Data was tabulated in a Microsoft Office EXCEL 2007 spreadsheet and transferred for analysis in SPSS 18.0. Quantitative variables were described as average and standard deviation average \pm SD and qualitative variables are presented in absolute frequencies and percentages. The sample showed a population of females $100 \%$ with an average age of $73.2 \pm 8,14$. Statistical analysis showed that $60 \%$ of them have two or more pathologies. Concerning to the congnition, $100 \%$ related having good cognition. Regarding the ability to perform activities of daily living, all $100 \%$ reported to be independent to perform these activities. The elderly women affiliated to a nursing home presented satisfactory results regarding cognition and ability to perform daily living activities. The majority of them, though, presented comorbities.
\end{abstract}

Keywords: Epidemiology. Elderly women. Risk.

\footnotetext{
${ }^{1}$ Curso de Fisioterapia. Universidade Ceuma
}

\section{Autor para correspondência:}

José Newton Lacet Vieira. e-mail: newtonlacet@hotmail.com

End.: Av. Nova York, Quadra 5E, Casa 3, Centro Park, Bairro Araçagy, Cep: 65.110-000.

São Luis-MA. 


\section{Introdução}

Envelhecer é um processo natural que caracteriza uma etapa da vida do homem e dá-se por mudanças físicas, psicológicas e sociais que acometem de forma particular cada indivíduo. É uma fase em que ponderando sobre a própria existência, o indivíduo idoso conclui que alcançou muitos objetivos, mas também sofreu muitas perdas, das quais a saúde destaca-se como um dos aspectos mais afetados. ${ }^{1}$

No Brasil, são consideradas idosas as pessoas com mais de 60 anos. 0 aumento desta população é um fenômeno observado em diversos países, inclusive no Brasil. $O$ atual perfil epidemiológico das doenças crônicas que acometem essa faixa da população leva consequentemente ao aumento da improdutividade dos indivíduos e aumento dos custos governamentais. ${ }^{2}$

O Brasil hoje é um jovem país de cabelos brancos. Todo ano, $650 \mathrm{mil}$ novos idosos são incorporados à população brasileira, a maior parte com doenças crônicas e alguns com limitações funcionais. Em menos de 40 anos, passamos de um cenário de mortalidade próprio de uma população jovem para um quadro de enfermidades complexas e onerosas, típicas da terceira idade, caracterizado por doenças crônicas e múltiplas. ${ }^{3}$

Estudos populacionais realizados no país têm demonstrado que não menos que $85 \%$ dos idosos apresentam pelo menos uma doença crônica. ${ }^{4}$

Em geral, os idosos percebidos como frágeis são aqueles que apresentam riscos mais elevados para desfechos clínicos adversos, tais como: dependência, institucionalização, quedas, piora do quadro de doenças crônicas, doenças agudas, hospitalização, lenta ou ausente recuperação de um quadro clínico e morte. ${ }^{5}$

A prioridade na atenção à saúde dos idosos deve voltar-se para estratégias que possibilitem a vida mais saudável, além da monitorização de indicadores capazes de avaliar a morbidade, o impacto da doença e/ou incapacidade na qualidade de vida dos idosos. ${ }^{6}$

De acordo com Costa et al. ${ }^{7}$, quanto à morbimortalidade, observa-se uma carga dupla de doenças. Por um lado, o perfil da mortalidade aproxima-se do observado em países desenvolvidos, com predomínio das doenças cardiovasculares e das neoplasias como primeira e segunda causa de óbito $31 \%$ e $17 \%$ do total, respectivamente. Por outro lado, persistem algumas doenças infecciosas e parasitárias e observa-se o surgimento de novas epidemias, como a Síndrome da imunodeficiência adquirida AIDS, ou o ressurgimento de outras, como a dengue e as leishmanioses em áreas urbanas.

É de grande valor e fundamental importância à sociedade e aos profissionais da área de saúde saber identificar as enfermidades que mais afetam esta população, uma vez que se trata de um grupo de risco para muitas doenças. Tendo em vista essa problemática, o presente estudo teve como objetivo descrever o perfil epidemiológico de idosas vinculados a um clube de repouso, para desta forma atuar eficazmente sobre 0 adequado nível de prevenção.

\section{Materiais e métodos}

Tratou-se de um estudo descritivo e transversal ${ }^{8}$, realizado no período de março a junho de 2013. O local da pesquisa foi o Clube de Repouso Irmã Dulce na cidade de São Luis - MA Brasil. A população deste estudo foi composta por conveniência por 30 idosas, com idade igual ou superior a 60 anos, vinculados ao Clube de Repouso Irmã Dulce.

Cada idosa participante assinou o Termo de Consentimento Livre e Esclarecido da Idosa, conforme a 
Resolução do Conselho Nacional de Saúde no $466 / 12^{9}$ sendo aprovado pelo Comitê de Ética e Pesquisa em Humanos (CEP), da universidade CEUMA, com parecer de nํ 289.169/2013.

Para o levantamento do perfil epidemiológico das idosas foi realizada uma entrevista adaptada por Danilow et al. ${ }^{10}$, que permitiu verificar as doenças mais prevalentes do grupo de idosas envolvidas na pesquisa além de domínios vinculados a aspectos correlacionados a saúde mental, sensorial, físico, social, nutricional e assistência terapêutica.

Foram verificadas as doenças mais prevalentes e a capacidade de compreensão e leitura através de entrevista individual.

Os dados foram tabulados em uma planilha do programa Microsoft Office EXCEL 2007, e transferidos para análise no programa estatístico SPSS 18.0. As variáveis quantitativas são descritas em média e desvio padrão média \pm DP e as variáveis qualitativas são apresentadas por meio de frequências absolutas e percentuais.

\section{Resultados}

A idade média das idosas foi de $73,2 \pm 8,14$ anos. Dentre as participantes, $60 \% \mathrm{n}=18$ apresentaram duas ou mais patologias. Nas que apresentaram apenas uma doença, $16,6 \% \quad n=5$ relataram hipertensão, $3,4 \% n=1$ diabetes mellitus e semelhante percentual relataram problemas relacionados ao sistema digestivo sem especificação da mesma.

No que se diz respeito a capacidade de realização de algumas atividades relacionadas a vida diária das idosas participantes da pesquisa, todas $100 \%$ relataram independência com relação a alimentação, vestuário e deambulação.

As informações obtidas sobre a saúde mental, sensorial, física, social, nutricional e à assistência terapêutica, descreveram o perfil epidemiológico das pessoas idosas vinculadas ao clube de repouso Irmã Dulce. Observou-se níveis satisfatórios na saúde destas idosas, excetuando-se 0 aspecto sensorial relacionado a visão Tabela 1.

\section{Discussão}

No que se diz respeito às patologias mais comuns encontradas nas idosas avaliadas, o presente estudo apontou como patologias mais comuns as doenças crônicas não transmissíveis. Atualmente no Brasil, com o aumento da longevidade das pessoas idosas houve paralelamente um aumento das doenças crônicas. Corroborando com o presente estudo, Santos et al. ${ }^{11}$ avaliaram 371 idosos, identificando como problemas de saúde mais comuns a hipertensão $58 \%$ e a artrite/artrose $31 \%$. Essa associação deve-se ao fato de que as mulheres possuem uma maior expectativa de vida e consequentemente maior risco de desenvolver doenças crônicas incapacitantes.

O presente estudo verificou que dentre as 30 idosas $100 \%$, todas apresentaram capacidade de desenvolver algumas atividades da vida diária AVDs, como se alimentar, vestir e andar. Uma maior independência funcional depende muito de não haver a prevalência de doenças em pessoas idosas. Nossos resultados estão de acordo com o estudo desenvolvido por Costa et al. ${ }^{7}$, onde participaram 116 indivíduos com idade igual ou superior a 60 anos e desses idosos 57,9\% eram independentes ao realizar as AVDs.

Contradizendo a esses dados, os achados do estudo realizado por Araújo e Ceolim $^{12}$, no qual detectaram que dentre 187 idosos, 70 encontravam-se independentes para desenvolver as AVDs, confirmando que, na maioria dos casos, a institucionalização está associada à dependência física e cognitiva. Uma possível explicação para esses achados discordantes pode ser 0 
Tabela 1. Aspectos do perfil epidemiológico de idosos vinculados a um clube de repouso em São Luís - MA, 2013

\begin{tabular}{|c|c|c|}
\hline & $\mathbf{n}$ & $\%$ \\
\hline \multicolumn{3}{|l|}{ Aspecto mental } \\
\hline \multicolumn{3}{|l|}{ Estado mental } \\
\hline Consciente & 29 & 96,7 \\
\hline Orientado & 1 & 3,3 \\
\hline \multicolumn{3}{|l|}{ Aspecto sensorial } \\
\hline \multicolumn{3}{|l|}{ Fala } \\
\hline Normal & 29 & 96,7 \\
\hline Arrastada & 1 & 3,3 \\
\hline \multicolumn{3}{|l|}{ Audição } \\
\hline Normal & 23 & 76,7 \\
\hline Prejudicada & 5 & 16,7 \\
\hline Surdez & 2 & 6,7 \\
\hline \multicolumn{3}{|l|}{ Visão } \\
\hline Normal & 11 & 36,7 \\
\hline Prejudicada & 19 & 63,3 \\
\hline \multicolumn{3}{|l|}{ Aspecto físico } \\
\hline \multicolumn{3}{|l|}{ Marcha } \\
\hline Normal & 24 & 80,0 \\
\hline Patológica & 6 & 20,0 \\
\hline \multicolumn{3}{|l|}{ Queda } \\
\hline Sim & 6 & 20,0 \\
\hline Não & 24 & 80,0 \\
\hline \multicolumn{3}{|l|}{ Aspecto social } \\
\hline \multicolumn{3}{|l|}{ Visitas } \\
\hline Família & 10 & 33,3 \\
\hline Amigos & 1 & 3,3 \\
\hline Outros & 1 & 3,3 \\
\hline Família e amigos & 12 & 40,0 \\
\hline Família, amigos e outros & 6 & 20,0 \\
\hline \multicolumn{3}{|l|}{ Aspecto nutricional } \\
\hline 3 refeições & 17 & 56,7 \\
\hline 4 refeições & 6 & 20,0 \\
\hline 5 refeições & 5 & 16,7 \\
\hline Nenhum & 2 & 6,7 \\
\hline \multicolumn{3}{|l|}{ Assistência terapêutica } \\
\hline \multicolumn{3}{|l|}{ Fisioterapia } \\
\hline Sim & 13 & 43,3 \\
\hline Não & 17 & 56,7 \\
\hline
\end{tabular}


local de origem da amostra e o número da mesma, visto que, o único grupo de idosas avaliadas foi de convivência, e a entrevista realizada não explorou muitos aspectos relacionados a capacidade destas pessoas de realizarem um maior número de AVDs.

No estudo exploratório e descritivo desenvolvido por Araújo e Coelim $^{12}$, foram avaliados idosos com idade igual ou superior a 60 anos em três instituições diferentes, ou seja, três amostras diferentes, na cidade de Taubaté. Já neste estudo foram avaliadas idosas vinculadas apenas a um clube de repouso em São Luís, o qual mostrou que estas pessoas participantes apresentaram uma independência funcional em algumas AVDs. Isto provavelmente ocorreu devido à boa participação delas nas atividades em grupo de forma ativa.

Em concordância com o estudo realizado, a pesquisa de seguimento de Rosa et al. ${ }^{13}$, enfatizou a ideia de que uma vida ativa pode ser capaz de manter por mais tempo a capacidade funcional do que a inatividade. Portanto, atividades aparentemente corriqueiras, como fazer ou receber visitas e frequentar a igreja, identificados nesses estudos como fatores associados com a capacidade funcional, tornam-se muito importantes e parecem traduzir a ideia de que a vida ativa é capaz de contribuir para a manutenção da capacidade funcional.

Del Duca et al. $^{14}$ afirmaram que o grande desafio para a saúde pública nas próximas décadas está no diagnóstico e prevenção dos possíveis riscos associados à incapacidade funcional, em busca de uma longevidade com maior independência, autonomia e qualidade para os idosos.

Em relação à capacidade de leitura e compreensão, todas 100\% relataram ser capazes de compreender, mas quase a metade $46,7 \%$ não consegue ler, daí porque a necessidade de ter sido realizada uma entrevista com as pessoas idosas, como forma de se obter uma informação mais fidedigna da saúde delas, sendo considerado que a maioria delas referiu ter a visão prejudicada Tabela 1.0 estudo realizado por Luíz et al. ${ }^{15}$ afirmaram que o déficit visual é um importante fator associado à dificuldade de realizar atividades cotidianas.

Neste estudo foi verificado que entre as 30 idosas participantes, 18 idosas $60 \%$ não eram alfabetizadas, o que também foi determinante para a realização de uma entrevista com elas. A taxa de analfabetismo é um dos indicadores de saúde importantes quanto ao aspecto social. Em um estudo conduzido por Soares et al. ${ }^{16}$, observou-se que, quanto maior a faixa etária menor a escolaridade, predominando entre as mulheres idosas de 60-70 anos de idade. É possível que o maior grau de escolaridade entre as idosas mais jovens tenha relação com os avanços ocorridos nos níveis de educação da população brasileira, pois entre 1940 e 2000 houve um aumento significativo na proporção de idosos alfabetizados.

Segundo Brito e Pavarini ${ }^{17}$, o predomínio de analfabetos entre os idosos avaliados é um dado sociodemográfico relevante, uma vez que a chance de ter dependência moderada/grave é cerca de 4 a 5 vezes maior entre os idosos com nível mais baixo de escolaridade.

Em relação a quedas, foi detectado que apenas $20 \%$ das 30 idosas sofreram algum tipo de queda nos últimos seis meses. Isso pode ser explicado pelo fato de que as idosas avaliadas neste estudo mostraram conseguir desenvolver uma boa independência funcional em algumas AVDs. Sabe-se que o idoso sedentário tem maior risco de sofrer quedas, quando comparados com idoso que 
pratica algum tipo de atividade física. Corroborando com nossos resultados, Pimentel e Scheiche ${ }^{18}$ afirmaram que os idosos que requerem ajudam para a realização das AVDs têm 14 vezes maior probabilidade de cair, quando comparados àqueles de mesma faixa etária, porém independentes.

\section{Conclusão}

Foi possível com este estudo conhecer importantes informações capazes de descrever o perfil epidemiológico das idosas vinculadas ao clube de repouso Irmã Dulce pela verificação dos aspectos: mental, sensorial, físico, social, nutricional e terapêutico para a identificação de fatores que influenciam na saúde destas pessoas.

Tendo em vista que a capacidade do ser humano declina com a idade, o presente estudo evidenciou descrever alterações da saúde, de ordem física, mental e social, que comprometam uma maior longevidade de idosas.

Contudo, mediante a amostra pequena de idosas disponíveis para este estudo, sugere-se que outros estudos sejam realizados com uma maior população, para que se tenha uma maior referência epidemiológica sobre idosas vinculadas a um clube de repouso.

\section{Referências}

1. Mendes MRSSBM, Gusmão JL, Faro $A C M$, Leite RCBO. A situação social do idoso no Brasil: uma breve consideração. Acta Paul Enferm. 184:422-6, 2005.

2. Gois ALB, Veras RP. Informações sobre a morbidade hospitalar em idosos nas internações do Sistema Único de Saúde do Brasil. Ciênc Saúde Coletiva. 2010;156:28-68.

3. Tannure MC, Alves $M$, Sena RR, Chianca TC. Epidemiological profile of the elderly population in Belo Horizonte, MG, Brazil. Rev Bras Enferm. 2010;635:817-22.

4. Silvestre JA, Costa Neto CMM. Abordagem do idoso em programas de saúde da família. Cad Saúde Pública. 2004;193:839-47.

5. Macedo C, Gazzola JM, Najas M. Síndrome da fragilidade no idoso: importância da fisioterapia: [revisão]. Arq Bras Ciênc Saúde. 2008;333:17784.

6. Victor JF, Ximenes LB, Almeida PC, Vasconcelos FF. Perfil sociodemográfico e clínico de idosos atendidos em Unidade Básica de Saúde da Família. Acta Paul Enferm. 2009;221:49-54.

7. Costa CE, Nakatani KYA, Bachion MM. Elder's community capacy to develop Daily Life Activities and Daily Instrumental Life Activities. Acta Paul Enferm. 2006;191:35-45.

8. Thomas JR, Nelson JK, Silverman SJ. Métodos de pesquisa em atividade física. Porto Alegre: Artmed; 2007.

9. Conselho Nacional de Saúde. Resolução no 196, de 10 de outubro de 1996. Aprova as diretrizes e normas regulamentadoras de pesquisas envolvendo seres humanos. Diário da República Federativa do Brasil, 16 mar 1996.

10. Danilow MZ, Moreira ACS, Villela CG, Barra BB, Novaes MRCG, Oliveira MPF. Perfil epidemiológico, sociodemográfico e psicossocial de 
idosos institucionalizados do Distrito Federal. Comun Ciênc Saúde. 2007;181:9-16.

11. Santos KA, Koszuoski R, Dias-daCosta JS , Pattussi MP. Fatores associados à incapacidade funcional entre os idosos em Guatambu, Santa Catarina, Brasil. Cad Saúde Pública. 2007;2311:2781-8.

12. Araújo HPM, Ceolim FM. Assessment of the level of independence of elderly residents in long-term care instituitions. Rev Esc Enferm USP. 2007;413:378-85.

13. Rosa, TEC, Benício MHD, Latorre MRDO, Ramos LR. Fatores determinantes da capacidade funcional entre idosos. Rev Saúde Pública. 2003;376:40-8.

14. Del Duca GF, Silva MC, Hallal PC. Disability relating to basic and instrumental activities of daily living among elderly subjects. Rev Saúde Pública. 2009;435:796- 805.

15. Luíz LC, Rebelatto JR, Coimbra AMV, Ricci NA. Association between visual deficit and clinical-functional characteristics among communitydwelling older adults. Rev Bras Fisioter. 2009;135:44-50.

16. Soares MBO, Tavares DMS, Dias FA, Diniz MA, Geib S. Morbidades, capacidade funcional e qualidade de vidade mulheres idosas. Esc Ana Ney. 2010;144:705-11.

17. Brito PRT, Pavarini LCS. The relationship between social support and functional nacity in el derly persons with cognitive alterations. Rev Latino-Am Enferm. 2012;204:677-84.

18. Pimental MR, Scheicher ME. Comparação do risco de queda em idosos sedentários e ativos por meio da escala de equilíbrio de Berg. Fisioter Pesqui. 2009;161:6-10. 